\title{
Optimization of Interaural Intensity Difference based Binaural Sonar Sensing System for Object Detection
}

\author{
Payman Rajai, Mohammed Jalal Ahamed \\ MicroNano Mechatronics Laboratory, Mechanical, Automotive and Materials Engineering Department, \\ University of Windsor \\ Windsor, ON, Canada \\ email: jahamed@uwindsor.ca
}

\begin{abstract}
Interaural Intensity Difference (IID) in binaural sonar systems is used for echolocation and obstacle sensing. In this article, we show by simulation the optimized system's parameters in terms of frequency, sensor separation distance and the working range for an IID based binaural sonar sensing system. Our result shows that the best performances with a frequency range between 100 to $300 \mathrm{kHz}$ and a separation distance, depending on the size of the microphone, in our case between $2 \mathrm{~cm}$ to $5 \mathrm{~cm}$ within the working range of $1 \mathrm{~m}$. The approach developed in this paper could be useful for mobile localization and mapping, particularly in compact size mobile devices.
\end{abstract}

Keywords-component; acoustic sensor, binaural system, Interaural Intensity Difference.

\section{INTRODUCTION}

Ultrasonic sensors are robust and low-cost, well suited for identifying objects/obstacles within a few meters, typically configured with an array of emitters and receivers to achieve an accurate mapping of the surrounding environment. Binaural ultrasonic sensors are inspired by natural hearing systems where two ultrasonic receivers flank an ultrasonic emitter. In a binaural ultrasonic the object's distance and bearing angle can be estimated from the differences in interaural time [1]-[5], amplitude [6], intensity [7]-[9] and/or phase [10], [11]. It is known that interaural time and phase difference are appreciable with low-frequency sounds. With high-frequency sounds, the interaural difference in amplitude or intensity is more convenient. For instance, bat's echolocation system uses Interaural Intensity Difference (IID) at two different frequencies as the cue to the direction of a prey [12].

In a binaural system, the degree of accuracy depends on the separation distance between the two receivers for interaural time difference cue. The sonar separation must be much larger than the wavelength of the sonic wave in use. The separation distance is usually 10 's of centimeters to 10 's of meters [13]. When the separation between the receiver microphones is large compared to the object's distance, there is a risk that one of the receivers does not pick up the signal. If the microphones are too close to each other (similar to miniature animals with little separation between the two ears), both microphones record essentially the same acoustic signal. This results in very small interaural amplitude and time cues, posing substantial challenges to directional sensitivity of the auditory system [14]. To amplify the interaural difference, fly-ear inspired acoustic sensors consisting of two wings mechanically coupled at the middle have been recently developed [15].

In this article, we will show by simulation that the approach using IID makes it possible to place the receivers as close together as their size permits and thus making small size sonar system where interaural time difference measurement is not convenient. Our binaural system consists of a single emitter scanning across the range of incident angle flanked by two receivers (Figure 1). In this paper, we develop a mathematical model relating systems parameters in an IID based binaural sonar sensing system. We later utilized the model to demonstrate that the sensor performance for object identification using IID is enhanced under certain frequency and separation range. In the next sections, we will establish the concept from a theoretical perspective, and subsequently, simulate results to evaluate the model.

\section{MATHEMATICAL FORMULATION}

The geometry of our binaural sonar system is depicted in Error! Reference source not found., which consists of a transmitter flanked by two fixed receivers. In the model, the transmitter and receivers are assumed to be of piston shape, with the transmitter located at the origin, and the two receivers at distance $d$ to its left and right sides. As illustrated in Fig. 1, the object coordinate with respect to the emitter placed at the center of the polar coordinate system is $(r, \beta)$, where $r$ is the radial distance and $\beta$ is the azimuth angle. The angular deviation is measured with respect to the normal line through the emitter, and it takes positive values in the clockwise direction. Likewise, the object coordinate with respect to the receiver is $\left(r_{r}, \beta_{r}\right)$. Note that $\beta_{r}$ is measured with respect to the line that passes through the center of the object. It is parallel to the emitter's normal and is positive in the clockwise direction. 


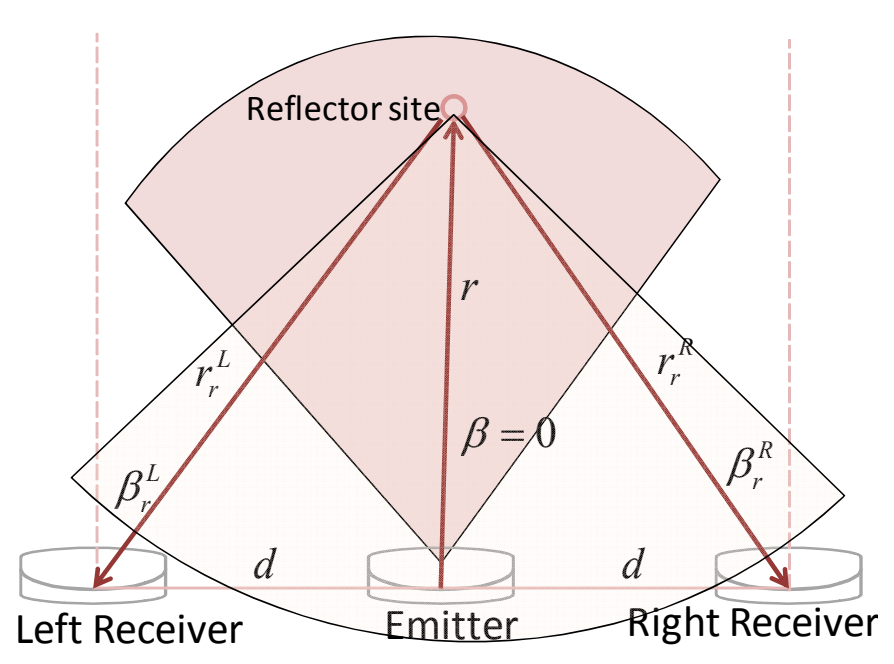

Figure 1. The geometry of the binaural sonar system with rotating emitter.

If a small object is ensonified by the emitter, it re-emits a spherical field that can be detected by a receiver. For pistonshaped emitter and receiver, the pressure field identified by the receiver can be described by [16], [17]

$$
\begin{aligned}
& P_{D}\left(k, r, \beta, r_{r}, \beta_{r}\right)=\frac{\rho c \sigma}{2} U_{0} \frac{k a_{e}^{2} a_{r}^{2}}{r_{e} r_{r}} \\
& \ldots \frac{2 J_{1}\left[k a_{e} \sin (\beta)\right]}{k a_{e} \sin (\beta)} \cdot \frac{2 J_{1}\left[k a_{r} \sin \left(\beta_{r}\right)\right]}{k a_{r} \sin \left(\beta_{r}\right)},
\end{aligned}
$$

where $a_{e}$ and $a_{r}$ are, respectively, the radius of the circular emitter and of the receiver surface, $k$ is the wavenumber relates to the frequency via $2 \pi \mathrm{f} / \mathrm{c}, c$ is the speed of sound, $\rho$ is the air density, $J_{1}$ is the Bessel function of the first kind, and $\sigma$ is the scattering coefficient, the ratio of the scattering and the incident fields. As depicted in Figure 1., the position of the object to the left or right of the receivers is obtained from

$$
r_{r}^{L, R}=\sqrt{d^{2}+r^{2}-2 d r \cos (\pi / 2 \pm \beta)}
$$

and

$$
\beta_{r}^{L, R}=\left|\sin ^{-1}\left(\frac{d \cos (\beta)}{r_{r}^{L, R}}\right) \pm \beta\right|,
$$

where the superscriptions $\mathrm{L}$ and $\mathrm{R}$ denote the left and the right receivers also are shown in Figure 1. Once the echo is detected by both receivers on the two sides of the emitter, the Interaural Intensity Difference (IID) is measured from absolute difference of the detected intensities that is the squared of amplitudes defined by $\mathrm{Eq}(1)$.

\section{SIMULATION}

The theoretical model developed in the previous section is simulated by sweeping the parameters of the system to evaluate the performance of the model in detecting an obstacle. A typical example of the detected intensities and the IID is presented in Figure 2. . In this example plot, we set $a_{e}=a_{r}=4.25 \mathrm{~mm}, \mathrm{U}_{0}=1000, r=30 \mathrm{~cm}, d=5 \mathrm{~cm}$ at $50 \mathrm{kHz}$ frequency.

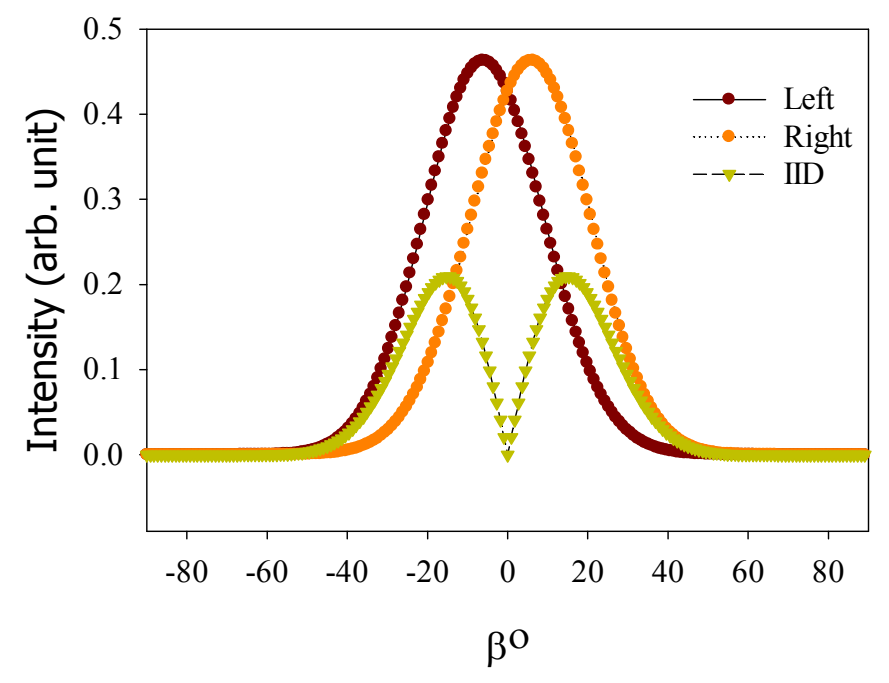

Figure 2. The detected intensities by the left and the right microphones and the IID.

In Figure 2. a point object is scanned for a range of azimuth angle from $-\pi / 2$ to $+\pi / 2$. It is observed that the intensity captured by each microphone is largest when the object is just above the microphone. The quantity of our interest is the IID that is zero when the object is aligned with the sonar axis as the sensor scans the environment. We use this property of the IID to scan the environment searching for obstacles for various system's parameters as frequency, the separation distance between the emitter and the receiver and working range.

The crucial condition here is that the zero point of the IID must be differentiable from other parts of the signal. In other words, the contrast between the zero and the maximum of the IID signal and the slope of the curve from the maximum towards the zero point defines the performance of such system to identify the obstacle effectively. In the next section, we will evaluate necessary conditions for best performance including the system's parameters of frequency $(f)$, separation distance $(d)$ and working range $(r)$.

\section{A. Tuning system's parameters}

In this section, we identify optimal parameters for the emitter frequency $(f)$, the separation between the microphones and the emitter $(d)$, and the working range $(r)$. To realize this, various combinations of $f, d$, and $r$ were simultaneously explored while looking for systematic variations in the IID contrast. 


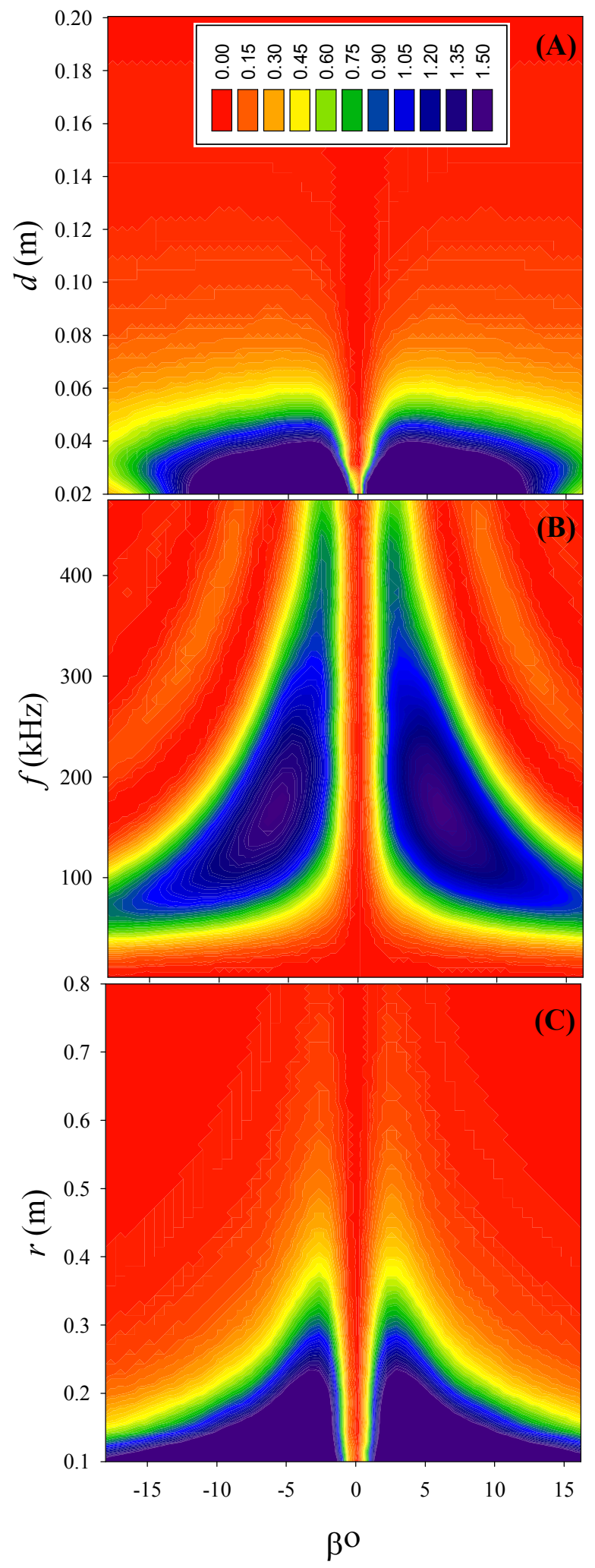

Figure 3. Color map of IID for a range of separation distance (A), frequency (B) and working range $(\mathrm{C})$ respectively.
The simulation test results for the IID variation with system's parameters are plotted in Figure 3 . where the horizontal axis corresponds to the scanning angle $\beta$. In Figure 3 . , red signifies low contrast, and blue denotes higher contrast desirable for better performance.

Figure 3. corresponds to the IID map with respect to varying separation distance between the emitter and the microphone $(d)$ that is swept from $2 \mathrm{~cm}$ to $20 \mathrm{~cm}$. From Figure 2. Figure 3. (A) it is observed that the best performance of the sonar system corresponds to the small separation between the emitter and microphones i.e. up to $6 \mathrm{~cm}$. The large separation will degrade the performance of the system especially with high-frequency ultrasonic waves where they are highly directive and therefore the IID contrast gradually fades away with increasing the separation distance. In other words, large separation is permissible with low-frequency source but will be impractical with high frequencies. This fact is shown in Figure 4. where the IID contour is plotted for a range of frequency versus separation distance. As the figure shows, with increasing the frequency, the separation distance that gives appreciable IID contrast would be limited to small distances.

In Figure 3. (B), the IID map is provided with respect to varying frequency from $1 \mathrm{kHz}$ up to $500 \mathrm{kHz}$. In this figure, the suitable frequency range is observed to be between 100 to $300 \mathrm{kHz}$ with the best performance around $200 \mathrm{kHz}$. The system is impractical for frequencies lower than $20 \mathrm{kHz}$ since the acoustic wave loses its directivity and the microphones record almost similar pressure field regardless of the separation distance. The system also works with frequencies higher than $300 \mathrm{kHz}$; however, side lobes appear in the recorded fields as well as the IID, which may make it difficult to distinguish the main IID zero from the side lobes. One possible approach with high-frequency emitter source is to reduce the separation distance as suggested in Figure 2. Figure 3. (A).

Regarding the working range of the system in Figure 2. Figure 3. (C), the radial distance from the emitter is swept from $10 \mathrm{~cm}$ up to $100 \mathrm{~cm}$. It is observed that the performance degrades with distance with the best performance correspond to distances lower than $50 \mathrm{~cm}$. This is also due to the ultrasonic natural degradation with distance. This limitation, however, can be easily overcome by increasing the emitter's power to generate stronger pressure fields. 


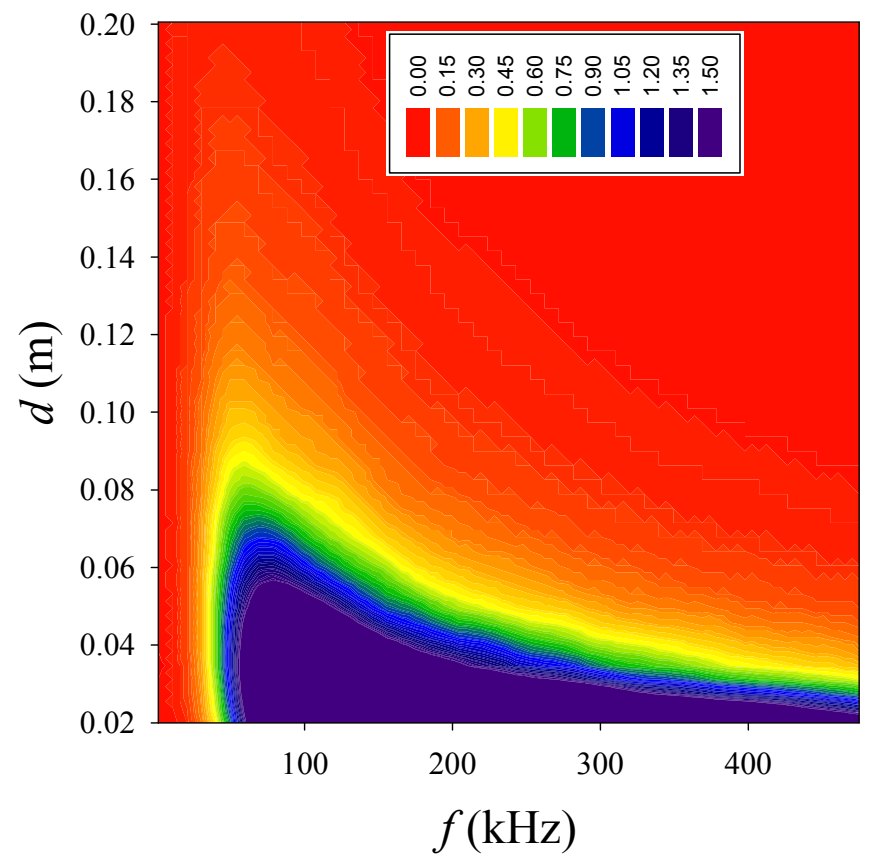

Figure 4. The IID contour for a range of frequency versus separation distance.

With respect to the above-findings, a suitable sonar system for working range within $1 \mathrm{~m}$ would consist of a transducer that emits ultrasonic pulses of $100 \mathrm{kHz} \leq f \leq 300 \mathrm{kHz}$, flanked by two microphones separated by $d<5 \mathrm{~cm}$.

\section{CONCLUSION}

In this paper, we presented the optimization of binaural sonar systems for object detection using IID measurement. This system as showed in this article is suitable for sonar systems where time of flight measurement is not practical. The simulation result showed that the performance of such system is enhanced with a frequency range between 100 to $300 \mathrm{kHz}$ with the best performance around $200 \mathrm{kHz}$ and the separation distance between the emitter and receiver up to maximum $6 \mathrm{~cm}$ within the working range of $1 \mathrm{~m}$.

\section{ACKNOWLEDGEMENTS}

The authors would like to thank Natural Sciences and Engineering Research Council of Canada (NSERC) and University of Windsor for financial supports.

\section{REFERENCES}

[1] B. Barshan and R. Kuc, "A bat-like sonar system for obstacle localization," IEEE Trans. Syst. Man Cybern., vol. 22, no. 4, pp. 636-646, Jul. 1992.

[2] R. Kuc, "Binaural sonar electronic travel aid provides vibrotactile cues for landmark, reflector motion and surface texture classification," IEEE Trans. Biomed. Eng., vol. 49, no. 10, pp. 1173-1180, Oct. 2002.

[3] W.-Y. Mu, G.-P. Zhang, Y.-M. Huang, X.-G. Yang, H.Y. Liu, and W. Yan, "Omni-Directional Scanning
Localization Method of a Mobile Robot Based on Ultrasonic Sensors," Sensors, vol. 16, no. 12, Dec. 2016.

[4] R. Kuc, "Biomimetic sonar locates and recognizes objects," IEEE J. Ocean. Eng., vol. 22, no. 4, pp. 616624, Oct. 1997.

[5] C. Baumann, C. Rogers, and F. Massen, "Dynamic binaural sound localization based on variations of interaural time delays and system rotations," J. Acoust. Soc. Am., vol. 138, no. 2, pp. 635-650, Aug. 2015.

[6] H. Peremans, A. Walker, and J. C. T. Hallam, "3D object localisation with a binaural sonarhead, inspirations from biology," in Proceedings. 1998 IEEE International Conference on Robotics and Automation (Cat. No.98CH36146), 1998, vol. 4, pp. 2795-2800 vol.4.

[7] R. Kuc, "Sensorimotor model of bat echolocation and prey capture," J. Acoust. Soc. Am., vol. 96, no. 4, pp. 1965-1978, Oct. 1994.

[8] B. A. Edmonds and J. F. Culling, "Interaural correlation and the binaural summation of loudness," J. Acoust. Soc. Am., vol. 125, no. 6, pp. 3865-3870, Jun. 2009.

[9] Y. Zhang and B. A. Wright, "An influence of amplitude modulation on interaural level difference processing suggested by learning patterns of human adults," $J$. Acoust. Soc. Am., vol. 126, no. 3, pp. 1349-1358, Sep. 2009.

[10] M. I. Mandel and D. P. W. Ellis, "A probability model for interaural phase difference," ISCA Tutor. Res. Workshop Stat. Percept. Audit. SAPA2006 16 Sept. 2006 Pittsburgh PA, pp. 1-6, 2006.

[11] C. Faller and J. Merimaa, "Source localization in complex listening situations: Selection of binaural cues based on interaural coherence," J. Acoust. Soc. Am., vol. 116, no. 5, pp. 3075-3089, Nov. 2004.

[12] A. W. Mills, "Lateralization of High $\square$ Frequency Tones," J. Acoust. Soc. Am., vol. 32, no. 1, pp. 132-134, Jan. 1960.

[13] A. Saxena and A. Y. Ng, "Learning Sound Location from a Single Microphone," in Proceedings of the 2009 IEEE International Conference on Robotics and Automation, Piscataway, NJ, USA, 2009, pp. 43104315.

[14] D. Robert, R. N. Miles, and R. R. Hoy, "Directional hearing by mechanical coupling in the parasitoid fly Ormia ochracea," J. Comp. Physiol. A, vol. 179, no. 1, pp. 29-44, Jul. 1996.

[15] D. Wilmott, F. Alves, and G. Karunasiri, "Bio-Inspired Miniature Direction Finding Acoustic Sensor," Sci. Rep., vol. 6, p. 29957, Jul. 2016.

[16] "Sensorimotor model of bat echolocation and prey capture," J. Acoust. Soc. Am., vol. 96, no. 4, pp. 19651978 , Oct. 1994.

[17] R. Kuc, "Three-dimensional tracking using qualitative bionic sonar," Robot. Auton. Syst., vol. 11, no. 3, pp. 213-219, Dec. 1993. 